\begin{tabular}{|c|c|}
\hline Title & Spontaneous Degrafting of Weak and Strong Poly cationic Brushes in A queous Buffer Solutions \\
\hline Author(s) & Ko, Y eongun; Genzer, Jan \\
\hline Citation & $\begin{array}{l}\text { Macromolecules, 52(16), 6192-6200 } \\
\text { https://doi.org/10.1021/acs.macromol.9b01362 }\end{array}$ \\
\hline Issue Date & $2019-08-27$ \\
\hline Doc URL & http:/hdl.handle.net/2115/79126 \\
\hline Rights & $\begin{array}{l}\text { This document is the A ccepted Manuscript version of a Published Work that appeared in final form in Macromolecules, } \\
\text { copyright c A merican Chemical Society after peer review and technical editing by the publisher. To access the final } \\
\text { edited and published work see https://pubs.acs.org/doi } 10.1021 / \text { acs.macromol.9b01362. }\end{array}$ \\
\hline Type & article (author version) \\
\hline File Information & Spontaneous Degrafting of Weak and Strong Polycationic Brushes in A queous Buffer Solutions.pdf \\
\hline
\end{tabular}

Instructions for use 


\title{
Spontaneous degrafting of weak and strong polycationic brushes in aqueous buffer
} solutions

\author{
Yeongun $\mathrm{Ko}^{1}$ and Jan Genzer ${ }^{1,2, *}$ \\ ${ }^{1}$ Department of Chemical and Biomolecular Engineering, North Carolina State University \\ Raleigh, NC 27695-7905, USA \\ ${ }^{2}$ Global Station for Soft Matter, Global Institution for Collaborative Research and Education \\ (GI-CoRE), Hokkaido University, Japan
}

\begin{abstract}
Polymers grafted to substrates have traditionally been considered stable because of the covalent bonds that hold the polymers attached to the substrate. However, several recent reports have indicated that grafted polymers may detach from substrates under specific conditions. In this work we report on a systematic study of polymer degrafting involving polycationic brushes with different degrees of quaternization (DQ, mol \%), which have been incubated in three different buffer solu-

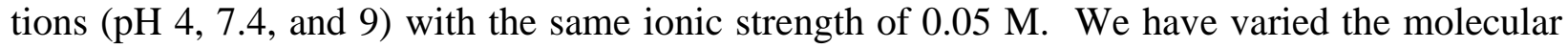
weight (MW) and grafting density $(\sigma)$ of the polymer brushes using a combinatorial set up to examine the effect of MW, $\sigma$, and DQ on polymer degrafting. Furthermore, we explored the effect of the bonding environment at the base of the initiator (mono- vs. tri-functional) of the grafted polymer layer at the substrate on the overall stability of polymer brushes on the substrate. The two major findings in this paper are: 1) degrafting of polycationic grafts from flat silica substrate increases with increasing DQ of the polymer, and 2) polymer degrafting likely occurs both in the initiator ester group and the silane head-group at the silicon substrate.
\end{abstract}

\footnotetext{
*Corresponding author: jgenzer@ncsu.edu
} 


\section{Introduction}

Polymer brushes feature polymer chains which are physically or chemically attached to a surface. ${ }^{1-}$

${ }^{3}$ Here, we consider chemically grafted polymer chains anchored to a flat impenetrable substrate.

The grafted polymers exhibit high stability and chains expansion in good solvents because every polymer chain is covalently anchored to the substrate. At low grafting density of polymer grafts, the spacing between grafted points on the substrate is larger than the radius of gyration $\left(\mathrm{R}_{\mathrm{g}}\right)$ of the grafted polymer. ${ }^{4}$ In this state (called "mushroom regime”) the polymer grafts do not interact with one another. At high grafting densities the distance between grafted points is much smaller than $\mathrm{R}_{\mathrm{g}}$ (called "brush regime”) and the polymers are sterically hindered by neighboring chains and swell in the direction normal to the substrate when incubated in good solvents. Polymer brushes with high grafting densities are typically prepared by the "grafting from” approach, which involves anchoring initiator molecules onto a solid substrate, followed by surface-initiated polymerization. ${ }^{1,3}$ Polymer brushes have been studied theoretically and experimentally for decades to understand and tune the properties of surfaces, including, anti-fouling, low-friction, responsive functions, etc. ${ }^{5-13}$ Numerous researchers have focused on studying the swelling behavior of polymer brushes as a function of the brush molecular weight or grafting density in good solvents. ${ }^{14-19}$

Several researchers reported on degrafting of hydrophilic polymer brushes in aqueous solution $^{20,21}$ and even degrafting of hydrophobic polymers in organic solvents. ${ }^{22}$ Polymer brush degrafting involves a breakage of a covalent bond in the initiator linker with the assistance of a mechanical force due to swelling of the brush. ${ }^{23}$ Many researchers have attempted to comprehend breaking a covalent bond in grafted polymers to disclose the mechanism of chain breaking by considering click chemistry ${ }^{24}$, photo-cleaving ${ }^{25-27}$, sonication-induced chain scission $^{28,29}$, strong acid or base ${ }^{30-33}$, etc. ${ }^{34-37}$ In our own previous work, we considered degrafting of polymer brushes 
from silicon substrates using tetrabutylammonium fluoride (TBAF). The fluorine ions in TBAF selectively cleave the Si-O bonds, leading to degrafting. ${ }^{38-41}$ We classified this mode as "on-demand degrafting (ODD)”. In a series of studies, we reported that polyanionic brushes detached from substrates upon increasing $\mathrm{pH}$ of the solutions that induced strong swelling due to electrostatic charging and concurrent hydrolysis of ester bonds in the initiator. ${ }^{42-45}$ This is considered as “spontaneous degrafting (SD)". Here we explore the effects of charge density, molecular weight, and grafting density on degrafting of weakly and strongly charged polycationic grafts and discuss

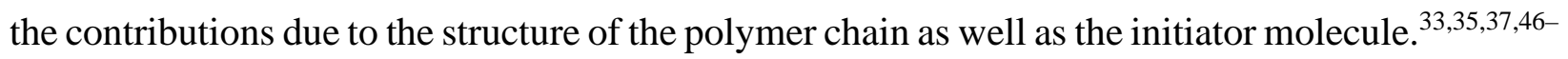
52

\section{Experimental section}

\section{Materials}

All chemicals were purchased from Sigma-Aldrich and used as received unless noted otherwise. Millipore Elix 3 was employed to obtain deionized water (DIW) with resistivity $>15 \mathrm{M} \Omega \cdot \mathrm{cm}$. 2-(dimethylamino)ethyl methacrylate (DMAEMA) was passed through an inhibitor removal column (Aldrich) before polymerization. 11-(2-bromo-2-methyl)propionyloxy undecyltrichlorosilane (tBMPUS) was purchased from Gelest. Silicon wafers (p-doped, orientation <100>) were purchased from Silicon Valley Microelectronics.

\section{Preparing polymer brushes}

Silicon wafers (14 mm x $40 \mathrm{~mm}$ in size) were exposed to ultraviolet/ozone treatment (UVO, Model 42, Jelight Co.) for 30 min before depositing the tBMPUS initiators from solution $(0.005 \% \mathrm{v} / \mathrm{v}$ of 
tBMPUS in HPLC grade toluene) at room temperature for 48 hours. 11-(2-bromo-2-methyl)propionyloxy undecylmonochlorodimethylsilane (mBMPUS) was prepared as described elsewhere. ${ }^{17}$ Briefly, 10 to 1 molar ratio of chlorodimethylsilane $(2.2 \mathrm{~mL})$ and 10-undecen-1-yl 2-bromo-2methylpropionate ( $0.704 \mathrm{~g}$ ) were placed in a dry vial with $20 \mu \mathrm{L}$ of Karstedt catalyst followed by nitrogen purging for overnight at room temperature. The mixture solution was kept under vacuum to remove excess reactants. The final product was stored as $0.005 \% \mathrm{v} / \mathrm{v}$ in HPLC grade toluene and used the same way as tBMPUS.

The PDMAEMA brushes were grown by surface-initiated atom transfer radical polymerization (ATRP). ATRP solution comprised molar ratios of [DMAEMA]:[CuCl]:[2,2'-bipyridine] of [60]:[1]:[2.3]. Specifically, DMAEMA (4 mL, $23.7 \mathrm{mmol}$ ), DIW (36.8 mL, $2.0442 \mathrm{~mol}$ ) and isopropanol $(9.2 \mathrm{~mL}, 120.3 \mathrm{mmol})$ were loaded into a $150 \mathrm{~mL}$ round-bottom flask followed by purging argon gas for 10 minutes. 2,2'-bipyridine $(0.1421 \mathrm{~g}, 0.9 \mathrm{mmol})$ and $\mathrm{CuCl}(0.03917 \mathrm{~g}, 0.4$ mmol) was added sequentially. The solution was mixed using a stir-bar and degassed by bubbling argon gas for another 15 mins. Surface-anchored PDMAEMA brushes were grown by incubating the initiator-coated substrates in a $20 \mathrm{~mL}$ vial filled with the ATRP solution for $120 \mathrm{mins}$ at room temperature. For poly(2-(methacryloyloxy)ethyl-trimethylammonium chloride) (PMETAC) brushes, the ATRP solution was prepared in molar ratios of [METAC]:[CuBr]:[2,2'-bipyridine] of [60]:[1]:[2.3]. METAC (11.46 mL, $60.1 \mathrm{mmol})$, DIW (3.9 mL, $0.2163 \mathrm{~mol})$ and isopropanol (14.6 $\mathrm{mL}, 0.1916 \mathrm{~mol}$ ) and were added into the reaction flask and treated using same procedure as the PDMAEMA ATRP solutions.

Quaternization of PDMAEMA brushes was carried out by immersing PDMAEMA brushes into a solution comprising $10 \mathrm{vol} \%$ of iodomethane in ethanol at room temperature for various reaction times. qPDMAEMA samples with degrees of quaternization (DQ) of 79, 71 and $79 \mathrm{~mol}$ 
\% were acquired by reacting PDMAEMA brushes with iodomethane for 45, 90, and 300 seconds, respectively. We use the nomenclature qPDMAEMA-DQx to refer to such samples, where $\mathrm{x}$ denotes the degree of quaternization (i.e., mole percent of quaternized qDMAEMA units in P(DMAEMA-co-qDMAEMA) copolymers).

A molecular weight gradient of PDMAEMA brushes was fabricated by vertically and stepwise dipping the initiator-deposited substrate into the ATRP solution (every $7 \mathrm{~mm}$ dip for $35 \mathrm{~min}$ of polymerization time) at room temperature. A grafting density gradient of PDMAEMA brushes was generated by vertically dipping the initiator-deposited substrate in $0.1 \mathrm{M}$ tetrabutylammonium fluoride (TBAF) in dimethylacetamide (DMA) solution in a step-wise fashion (every $7 \mathrm{~mm}$ dip for 3 min of TBAF degrafting time) at $50{ }^{\circ} \mathrm{C}$ followed by rinsing with methanol. The prepared substrate was then placed in a vial filled with ATRP solution for 120 mins. Figure S1 plots the corresponding film thickness profiles of the molecular weight and grafting density brush gradients.

\section{Preparing buffered solutions}

Solutions of $\mathrm{pH} 4,7.4$, and 9 were prepared with acetic acid, tris(hydroxymethyl)aminomethane, and ethanolamine buffer, respectively and $\mathrm{pH}$ was adjusted by adding $\mathrm{HCl}$ and $\mathrm{NaOH}$. The ionic strength was maintained at $0.05 \mathrm{M}$ with $\mathrm{NaCl}$ salts.

\section{Characterization}

We employed variable angle spectroscopic ellipsometry (VASE) (J.A. Woollam Co.) to measure the thickness and refractive index of polymer thin films. The ellipsometry data were analyzed using WVASE32 software (J.A. Woollam Co.). Every measurement was performed at two angles 
of incidence ( 60 and $65^{\circ}$ relative to the normal) between 400 and $800 \mathrm{~nm}$. A hot stage (FP82HT, Mettler Toledo) connected to a central processor (FP90, Mettler Toledo) was attached to the VASE sample stage. This assembly enabled controlling the temperature of polymer films during the ellipsometry measurements. Relative humidity was not controlled during the experiments; it was measured to be $\sim 50 \%$ at room temperature. We measured the thickness of PDMAEMA and qPDMAEMA at elevated temperatures $\left(100^{\circ} \mathrm{C}\right)$ to minimize water sorption. ${ }^{53}$ We used a single layer model to fit the ellipsometric data. This model comprises: $\mathrm{Si}$ substrate, $\mathrm{SiO}_{x}$ layer (thickness $1.5 \mathrm{~nm}$ ), and a Cauchy layer ( $n=A_{n}+B_{n} / \lambda^{2}$, where $\mathrm{n}$ is refractive index and $A_{n}$ and $B_{n}$ are fitting parameters).

The degree of quaternization (DQ, mol \%) was estimated by using the refractive index at $600 \mathrm{~nm}$. In our previous work, we demonstrated that DQ obtained from elemental analysis is linearly proportional to the refractive index which can be obtained from VASE measurements. ${ }^{53}$ The refractive index of pure PDMAEMA was measured and that of qPDMAEMA DQ-100 was estimated by extrapolation. Those values were used in the effective medium approximation (EMA). DQ (mol \%) could be obtained from DQ (vol \%) by using density and molecular weight of the polymers. We have used the following equation to relate $\mathrm{DQ}$ to $n: D Q=(835.10 \times n-$ 1233.77).

To measure the wet thickness of polymer brushes, we employed a liquid cell featuring fixed windows fixed at $70^{\circ}$ (relative to the vertical direction) for the incoming and outgoing light signals of the ellipsometer. All wet thicknesses were measured after 10 mins of incubation for each specimen in the liquid medium. We used a two-layer model to fit the ellipsometry data. This model comprises $\mathrm{Si} / \mathrm{SiO}_{x} /$ Cauchy1/EMA(Cauchy2 + water). The effective medium approximation (EMA) uses a volume-averaged layer combining the optical properties of the polymer and water. 
The Cauchy1 and Cauchy2 use fixed $A_{n}$ and $B_{n}$ values as 1.4686 and 0.005 , respectively. The thicknesses of Cauchy1 and EMA layer and the volume fraction of water in the EMA were used as the fitting parameters. Swelling ratio $(H / h)$ was obtained from wet thickness divided by dry thickness which measured at $100^{\circ} \mathrm{C}$. From our previous work, the difference in the "true" dry thickness (i.e., thickness at ambient conditions) for a polymer film having $\sim 100 \mathrm{~nm}$ thickness $<5$ $\mathrm{nm}$ (i.e., $<5 \%$ ) compared to the thickness measured at $100^{\circ} \mathrm{C} .{ }^{53}$

Fourier-transform infrared spectroscopy (FTIR) spectra were collected in attenuated total reflection (ATR) mode with Ge crystal on a Nicolet 6700 spectrometer and analyzed with OMNIC software. All FTIR spectra (shown in Figure S2 and S3) were collected by performing 128 scans with $4 \mathrm{~cm}^{-1}$ resolution.

\section{Results}

The dry thickness of polymer layer $(h)$ is related to the grafting density $\left(\sigma\right.$, chains $\left./ \mathrm{nm}^{2}\right)$ and the degree of polymerization $(N)$ through a simple mass balance given by:

$$
h=\frac{\sigma \cdot N \cdot M_{0}}{\rho \cdot N_{A}}
$$

where $M_{0}, \rho$ and $N_{A}$ represent the monomer molecular weight, polymer density, and the Avogadro's number, respectively. When exposed to a good solvent the polymer chains swell because of excluded volume interactions.

PDMAEMA polymers ( $\mathrm{pK}_{\mathrm{a}} \sim 7.5$ in bulk and $\mathrm{pK}_{\mathrm{a}} \sim 6.5$ for grafted chains) are neutral at $\mathrm{pH}$ 9 and fully charged in $\mathrm{pH} 4$ solution. ${ }^{18,19,54-57}$ Due to electrostatic interactions among the charged units the brushes at $\mathrm{pH} 4$ are more stretched than those at $\mathrm{pH} 9$. Thus, the swelling ratio $(H / h$, i.e., wet thickness normalized by the dry thickness) at $\mathrm{pH} 4$ is the highest and the one at $\mathrm{pH} 9$ is the

lowest (Figure 1a). We measured the variation of the swelling ratios with changing the degree of 
quaternization (DQ). At $\mathrm{pH} 4$ all the repeat units are protonated, having the highest swelling ratio regardless of DQ. At $\mathrm{pH}$ 9, the swelling ratio values increase with increasing DQ. At high DQ ( 80 mol \% in this study, except for PMETAC), the polymer chains are strongly stretched and possess similar swelling ratio values regardless of the $\mathrm{pH}$. At $\mathrm{pH} 7.4, \sim 50 \%$ of the repeat units are protonated. With increasing $\mathrm{DQ}$, the swelling ratio increases up to $\sim 4$, which is similar to the stretching ratio of PDMAEMA measured at $\mathrm{pH} 4$.

(a)

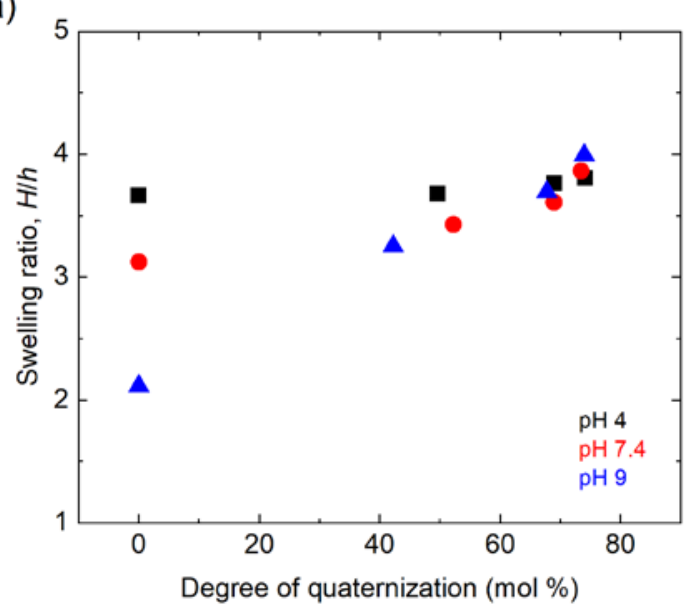

(b)

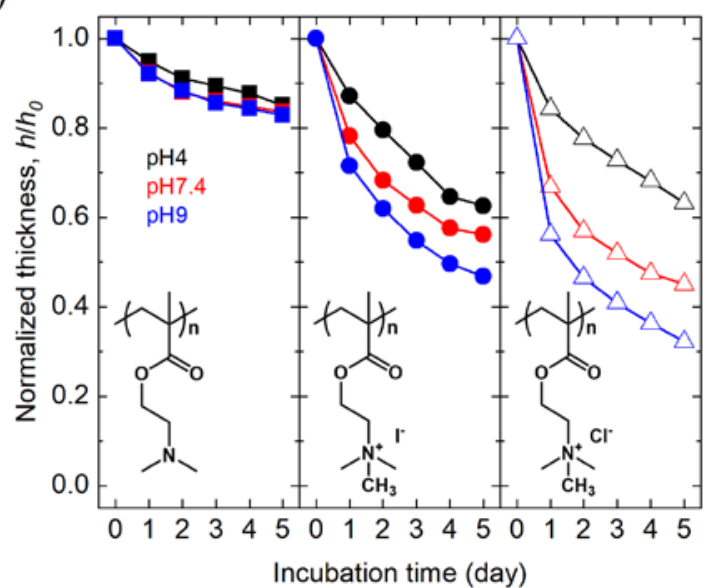

Figure 1. (a) Swelling ratio of qPDMAEMA brushes with different degree of quaternization (DQ) in solutions with variable $\mathrm{pH}$. The dry thickness of PDMAEMA brushes (before quaternization) is $92.8 \pm 0.8 \mathrm{~nm}$. (b) Measured normalized dry thickness of PDMAEMA brushes (left panel, solid symbols), and qPDMAEMA-DQ79 brushes (middle panel, solid symbols) and PMETAC brushes (right panel, open symbols) with incubation time in solutions with variable $\mathrm{pH}$. The lines are meant to guides the eye. The initial dry thickness values $\left(h_{0}\right)$ are: $98.3 \mathrm{~nm}$ (PDMAEMA), $122.4 \mathrm{~nm}$ (qPDMAEMA-DQ79), and $101.2 \mathrm{~nm}$ (PMETAC). All dry thickness data have been collected at $100^{\circ} \mathrm{C}$. The PMETAC brushes (open symbols in the right panel) may possess a different initial grafting density and molecular weight than the PDMAEMA and qPDMAEMA brushes.

The PDMAEMA, qPDMAEMA-DQ79, and PMETAC brushes were incubated in solutions having $\mathrm{pH} 4,7.4$, and 9 and a constant ionic strength of $0.05 \mathrm{M}$. Figure $1 \mathbf{b}$ displays the normalized dry thickness $\left(h / h_{0}\right)$ of each brush after incubation in solutions of various $\mathrm{pH}$ for various times. The brush thickness was measured at $100^{\circ} \mathrm{C}$ after removing each sample from the solution. In our previous report, we showed that the "true” dry thickness for hydrophilic polymer 
films can be obtained by performing ellipsometry measurements at $100^{\circ} \mathrm{C} ; 3$ at these conditions water molecules that solvate hydrophilic polymers (more pronounced for polyelectrolytes) should be removed from the sample. We stress that removing water from the film is important since water presence in the polymer layer influences of the overall brush thickness. In all cases we detect a decrease in brush thickness with increasing incubation time in the respective solution. This decrease in film thickness is driven by chain removal from the substrate due to hydrolysis of ester bonds in the initiator and/or the detachment of the silane from the substrate due to breakage of the Si-O bond. Thus, during degrafting the molecular weight of the brushes remains unchanged and the decrease in the normalized thickness is due to a decrease in the chain grafting density on the substrate. Incubating polymer brushes in the $\mathrm{pH} 9$ solution results in the largest amount of polymer degrafting, confirming base-catalyzed hydrolysis reaction. The $\mathrm{pH}$ effect on degrafting of PDMAEMA brushes is very small. The DMAEMA repeat units in this brush remain neutral at $\mathrm{pH}$ 9 but hydroxide ions may induce some degrafting. At $\mathrm{pH} 4$ most of the DMAEMA repeat units are positively charged (vide supra), thus inducing mechanical forces which act on the initiator linkages. ${ }^{18,19,54-56}$ However, at these solution conditions the hydrolysis of the ester and/or silane head-group is slow. In contrast, the degrafting of qPDMAEMA-DQ79 brushes shows a distinct dependence on $\mathrm{pH}$. Since the qPDMAEMA-DQ79 brushes were manufactured from same piece of parent PDMAEMA brush, both samples should have the same initial average grafting density $(\sigma)$ and degree of polymerization $(N)$. For qPDMAEMA-DQ79, 79 mol $\%$ of repeat units are charged even at pH 9 so that the initiators are susceptible to mechanical forces due to chain solvation and stretching. The hydroxide ions in $\mathrm{pH} 9$ can catalyze the hydrolysis at the initiators, resulting in severe degrafting. Although the brushes are heavily charged at $\mathrm{pH} 4$, the degrafting is lower than that in polymer brushes incubated in $\mathrm{pH}$ 9. We observe the same trend with PMETAC 
(qPDMAEMA-DQ100) brushes. The exposure of PMETAC brushes (albeit with a different counterion) to solutions of $\mathrm{pH} 9$ results in the highest degrafting among all cases studied. Assuming that PMETAC has a comparable $\sigma$ and $N$ as the PDMAEMA and qPDAEMA-DQx samples, the PMETAC's tendency to degraft the most among all samples studied, supports the notion of mechanical force induced degrafting mechanism at the initiator site due to strong chains swelling.

It is interesting to compare the results of PDMAEMA, qPDMAEMA-DQ79 and PMETAC brushes incubated in $\mathrm{pH}$ 4. The PMETAC and qPDMAEMA-DQ79 brushes degraft more strongly than PDMAEMA brushes even though they have nearly the same charge density. The degrafting of PMETAC brushes in $\mathrm{pH} 4$ is nearly identical to that of qPDMAEMA-DQ79. We attribute this behavior to the steric hindrance effect originating from the extra methyl group attached to the quaternary ammonium as well as the presence of the "bulky" counterions. This observation should be confirmed by additional experiments involving longer alkyl chain substituents (e.g., ethyl iodide or propyl iodide).

(a)

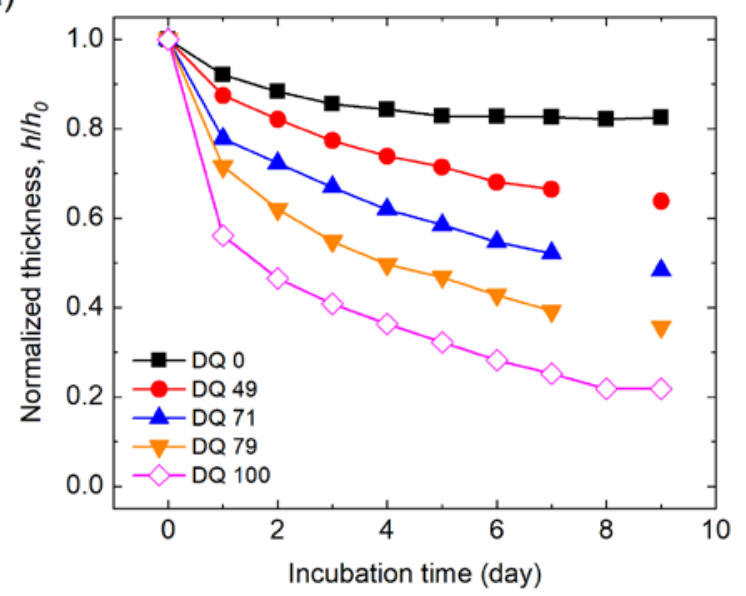

(b)

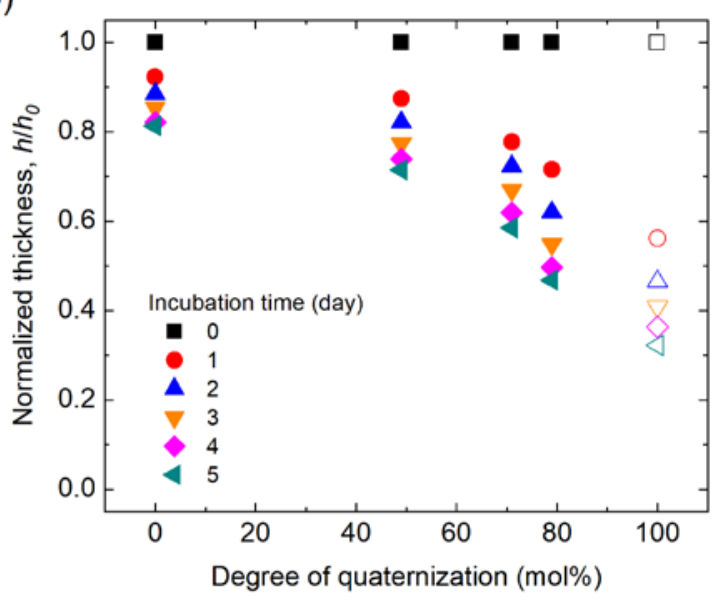

Figure 2. Normalized dry thickness of qPDMAEMA brushes measured at $100^{\circ} \mathrm{C}$ as a function of (a) incubation time. The lines are meant to guide the eye. (b) The same data set is represented as function of the degree of quaternization (DQ, in mol \%). The initial dry thickness values $\left(h_{0}\right)$ are: 98.3, 108.0, 115.5, 122.4, and $101.2 \mathrm{~nm}$ for DQ0, 49, 71, 79, and 100, respectively. All brushes have been incubated in $\mathrm{pH} 9$ solutions. The PMETAC brushes (open symbols) may have a different initial grafting densities $\left(\sigma_{0}\right)$ and $N$ relative to those of the PDMAEMA and qPDMAEMA brushes. 
From the result displayed in Figure 1, the mechanical force originating from brush swelling applied at the initiator site is a critical parameter that governs degrafting of polymers from the substrate. The mechanical force can be tuned by changing DQ, as demonstrated by the data plotted in Figure 2. All qPDMAEMA brushes (except perhaps for PMETAC brushes) possess the same average grafting density and degree of polymerization; the only difference among the samples is their DQ. The extent of degrafting and degrafting kinetics increase with increasing DQ. From Equation S5, the elastic force acting on each graft increases nonlinearly with increasing the wet thickness or increasing DQ. By plotting the normalized thickness as a function of DQ (Figure 2b) we find that the normalized thickness of the brushes decreases nonlinearly with increasing DQ.

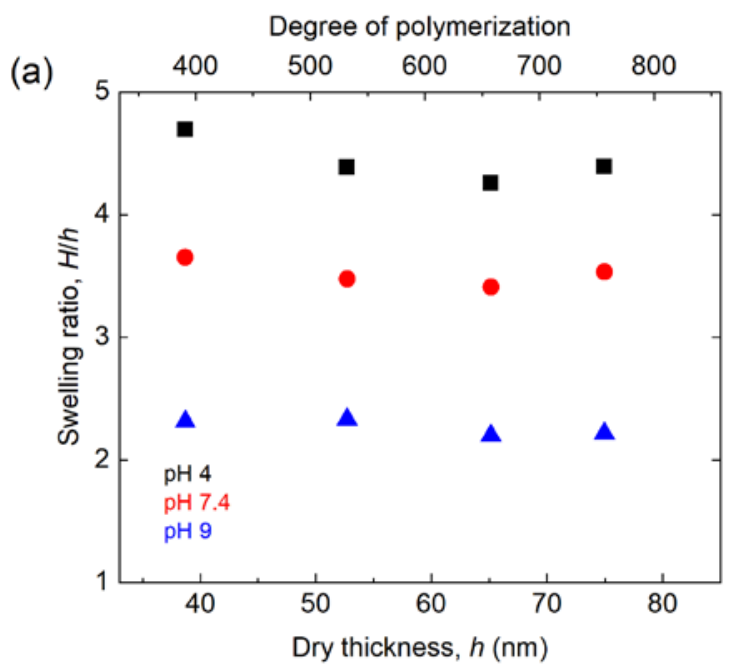

(b)

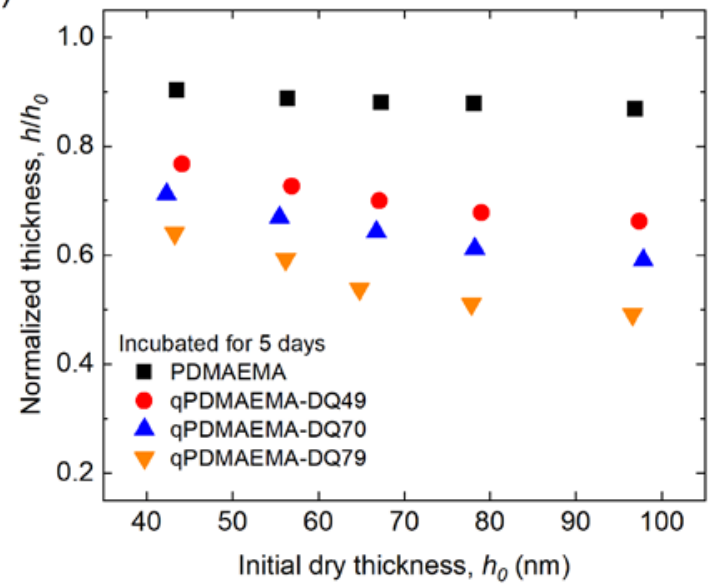

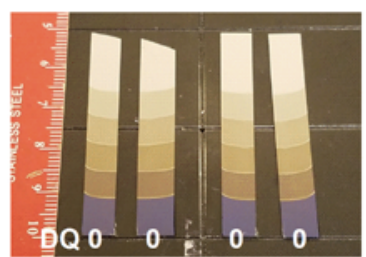

PDMAEMA brushes with MW gradient

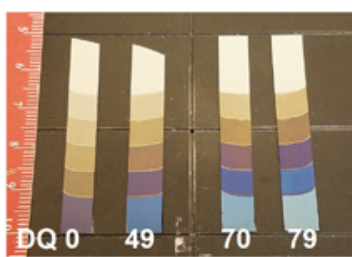

qPDMAEMA brushes with MW gradient

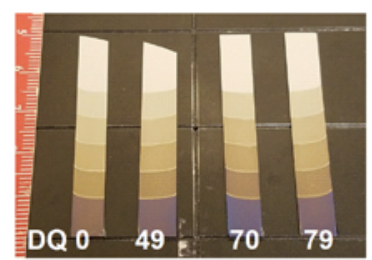

after 2 days incubation

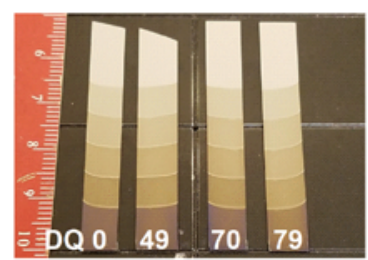

after 5 days incubation

Figure 3. (a) Swelling ratio of PDMAEMA brushes with different dry thickness (or degree of polymerization under the assumption of constant grafting density as 0.5 chains $/ \mathrm{nm}^{2}$ ). (b) Normalized dry thickness of qPDMAEMA brushes having different degrees of quaternization with molecular weight after incubating 5 days in $\mathrm{pH} 9$ solution. The photographs depict images of the actual samples. 
Samples featuring gradients in molecular weight (MW) of PDMAEMA were formed by vertically dipping of the initiator-deposited substrates in a stepwise fashion into the ATRP solution (see SI). The MW gradient PDMAEMA brushes were quaternized by reacting with 10 vol \% methyl iodide in ethanol for $0,45,90$, and 300 seconds to generate brushes with DQ of $0,49.1 \pm 3.1$, 69.9 \pm 1.2 , and $79.0 \pm 1.2 \mathrm{~mol} \%$, respectively. At a constant grafting density and $\mathrm{pH}$, increasing the chain length does not change the brush swelling ratio (vide supra and Equation S5-S9). According to Equation S5, there should be no change in mechanical force applied on the initiators. This means that the extent of degrafting should remain the same for brushes with different chain lengths. To confirm the scaling relationship, we measured the swelling ratio values on specimens featuring a gradient in MW (i.e., $N$ or dry thicknesses, $h$ ) of PDMAEMA (Figure 3a). All swelling ratio values are independent of $h$, proving that the gradient brushes have a comparable grafting density with increasing $\mathrm{MW}$ (or $N$ ). From our previous work, we assume the grafting density is $\sim 0.5$ chains $/ \mathrm{nm}^{2}$ so the degree of polymerization can be calculated from the Equation 1, plotted as top abscissa in Figure 3a. ${ }^{38,39}$

Figure 3b plots the normalized dry thicknesses after incubation for 5 days in solution having pH 9 with initial PDMAEMA brushes dry thickness ranging from 42 to $97 \mathrm{~nm}$. The bottom panel of the figure displays photographs of the actual samples. For PDMAEMA brushes, the extent of degrafting is nearly identical for all chain lengths, which agrees with the scaling result. However, for samples with DQ>0 the extent of degrafting increases slightly with increasing $N$ and DQ. We tentatively explain this behavior by considering the effect of dispersity in MW $(\nexists)$ of the brushes. There are only a few studies discussing the effect of $Ð$ on the conformation of polymer brushes, in which the authors have shown that the density profiles of the polymers changes from a 
parabolic concave to a linear to a convex shape when the $\oslash$ of the brushes changes from monodisperse to moderately disperse ( $(\sim 1.15)$ to highly disperse $(\nexists \sim 2)$, respectively. ${ }^{58-60}$ The density profile is derived from brush conformations, which, in turn, affects the elastic force acting on each chain. Thus, the elastic force may vary in brushes with different dispersity. For the surface-initiated ATRP, the longer chains may possess higher dispersity in MW. ${ }^{61,62}$

(a)

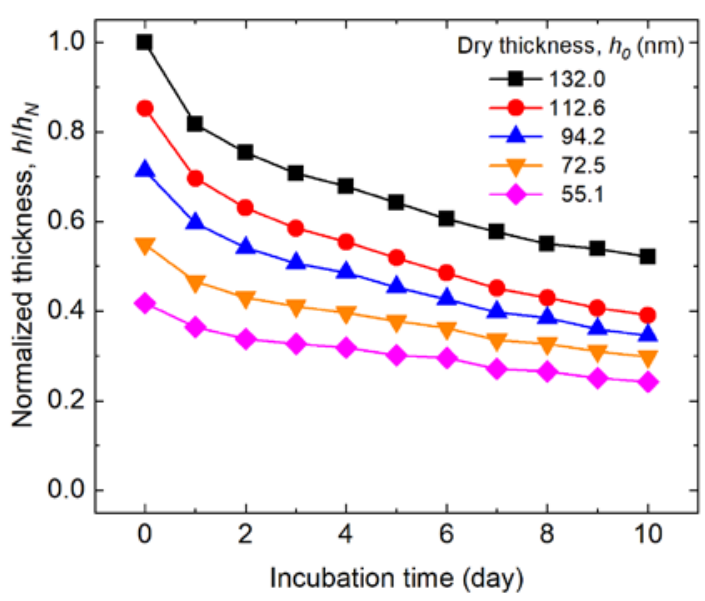

(b)

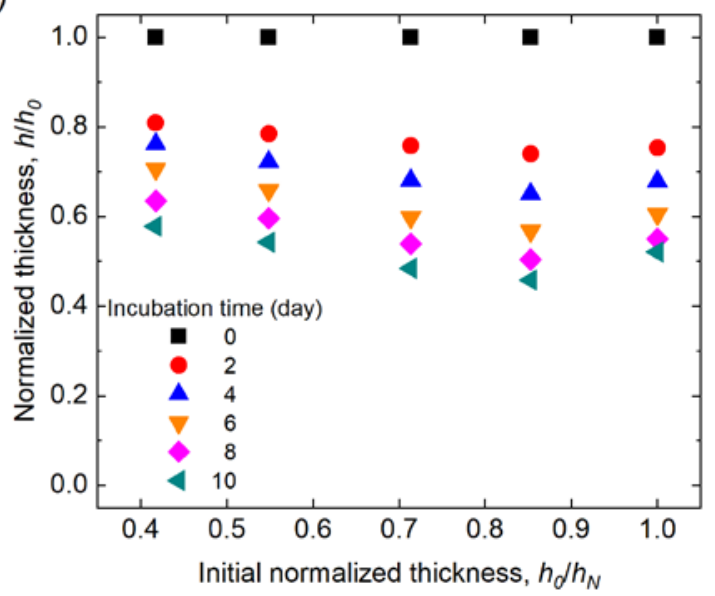

Figure 4. (a) Normalized dry thickness of qPDMAEMA-DQ82 brushes having different grafting densities as a function of the incubation time in $\mathrm{pH} 9$ solution. The legend indicates the initial dry thickness $\left(h_{0}\right)$ of qPDMAEMA-DQ82 before the incubation. All data were normalized by $132.0 \mathrm{~nm}\left(h_{N}\right)$, is the highest thickness of polymer brushes with the highest initial grafting density. The lines are meant to guide the eye. (b) The same data is represented as function of the initial normalized dry thickness of qPDMAEMA-DQ82 brushes. All data are normalized by initial dry thickness (i.e., from right to left data normalized by 132.0, 112.6, 94.2, 72.5, and $55.1 \mathrm{~nm}$, respectively).

We also prepared specimens featuring grafting density gradients of PDMAEMA and qPDMAEMA brushes. After forming a uniform self-assembled monolayer (SAM) of silane-based initiators on a silicon substrate, the sample was immersed vertically in a stepwise fashion into a solution featuring $0.1 \mathrm{M} \mathrm{TBAF}$ in DMA at $50^{\circ} \mathrm{C} .{ }^{40}$ After that, the substrate was placed in a vial filled with the ATRP solution to achieve a grafting density gradient of polymer brushes (see SI). This approach may lead to small variations in MW of the brushes due to the different confinement at different grafting densities. ${ }^{62}$ From our past experience this effect, if present, is negligible. 
However, since the extent of degrafting is nearly constant regardless of the MW variation as shown in Figure 3, we can assume here that the MW effect on degrafting is negligible (vide supra). In Figure 4a, we plot the dry brush data normalized by $132 \mathrm{~nm}$, which was the initial dry thickness of the sample in the highest grafting density region $\left(h_{0}\right)$. The data in Figure 4a reveal that the extent of degrafting increases with increasing incubation time in the $\mathrm{pH} 9$ solution. The relative extent of degrafting is higher in samples with higher initial grafting density. This is not surprising since densely grafted brushes are stretched away from the substrate due to excluded volume interactions with their neighbors and thus experience higher elastic force. ${ }^{33}$ The number of degrafted chains from the substrate decreases with decreasing polymer grafting density.

By normalizing the degrafting data by the corresponding initial dry thicknesses, we gain insight into the grafting density effect on degrafting ( $c f$. Figure $\mathbf{4 b}$ ). The results show only a small difference between the lowest and the highest grafting densities. Further analysis will be described below in Figure 7.

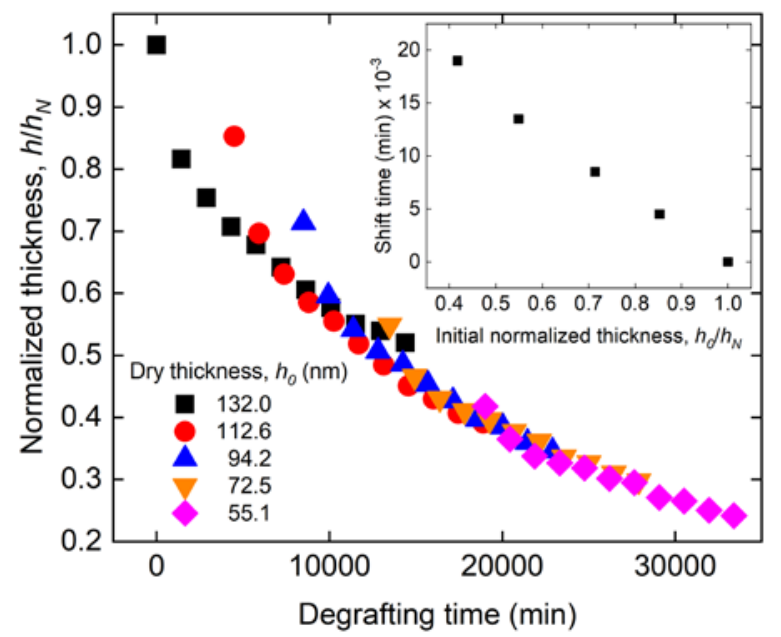

Figure 5. Normalized dry thickness of qPDMAEMA-DQ82 brushes having different grafting densities (from Figure 4a) as a function of the shifted degrafting time. The inset displays a linear relationship between shift time and initial normalized thickness. $\left(h_{N}=132 \mathrm{~nm}\right)$ 
The degrafting kinetics of grafting density gradient can be superimposed onto single master curve by horizontally shifting the data in Figure 4a (cf. Figure 5). The rational is that regardless of the initial grafting density the degrafting process should follow the same time dependence. In our analysis, we omitted the first degrafting point. In our previous paper we attributed the initial degrafting data to removing multilayers/imperfectly-bound chains present at the very surface that would be liberated first from the grafted polymer layer. ${ }^{41}$ Employing the “data-shifting” procedure brings all experimental data to a master curve. The inset in Figure 5 displays a linear dependence of the employed time shift as the as a function of the initial normalized grafting density of polymer brushes $\left(h_{0} / h_{N}\right)$.

Up until now, we used the tBMPUS as the initiator. This molecule features a tri-functional silane head-group that enables attachment to the substrate or/and neighboring silane molecules by up employing to 3 covalent bonds. To study the effect of the silane head-group structure on the stability of brushes, we prepared SAMs featuring mBMPUS as an initiator. mBMPUS features one functional unit and two methyl groups attached to the silicone head-group. This initiator should form a true monolayer on the supporting substrate. We grew PDMAEMA brushes from both the mBMPUS and tBMPUS in a vial filled with ATRP solution and quaternized them to obtain qPDMAEMA-DQ82 brushes. The mono-functional silane has a lower grafting density than the tri-functional silane because the mono-functional silane experiences steric hindrance from two methyl units in the silicone head-group. ${ }^{63,64}$ Unfortunately, we could not estimate either the degree of polymerization or the grafting density. However, we know that the effect of the degree of polymerization on degrafting is negligible for PDMAEMA and relatively small for qPDMAEMA (cf. Figure 3b). Figure 6 shows the results of degrafting PDMAEMA and qPDMAEMA-DQ82 from both mBMPUS- and tBMPUS-grown initiator centers. All data demonstrate that polymer 
brushes grown from mBMPUS experience larger extent of degrafting than those grown from tBMPUS. This result is very important as it shows that the silane head-group structure plays a crucial role in controlling the stability of polymers grafted to the substrate.

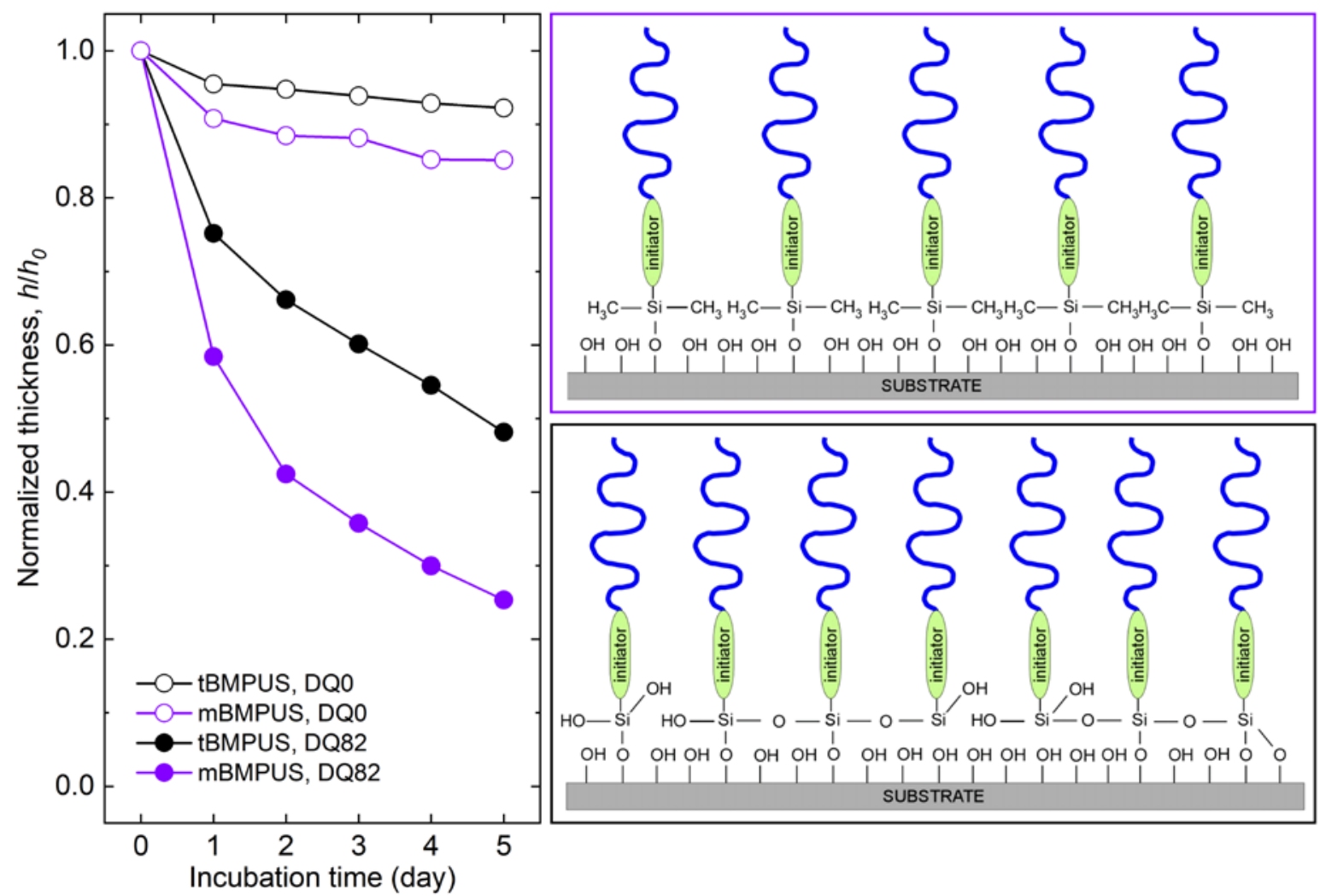

Figure 6. Normalized dry thickness of PDMAEMA (open) and qPDMAEMA-DQ82 (solid) brushes having different silane-based initiator structures with incubation time. The lines are meant to guide the eye. The initial dry thickness $\left(h_{0}\right)$ of mBMPUS based brushes were 47.7 and $60.2 \mathrm{~nm}$ for DQ0 and DQ82, respectively. The $h_{0}$ values of tBMPUS-based brushes were 93.4 and $119.8 \mathrm{~nm}$ for DQ0 and DQ82, respectively. The cartoons on the right describe a tentative structure of the tBMPUS (black border) and mBMPUS (violet border) initiators on the surface.

We can make a few conclusions from the experimental results discussed previously and from earlier publications. ${ }^{40,44,45,65-67}$ From the data in Figure 1, degrafting involves base-catalyzed hydrolysis that takes place in the initiator centers. The BMPUS initiators have two kinds of bonds that can be hydrolyzed: 1) ester/amide bond close to the initiating center, and 2) siloxane bond. Galvin and coworkers ${ }^{67}$ studied the effect of ester vs. amide in the BMPUS initiator on stability of 
polymer brushes and concluded that amide bonds are less prone to hydrolysis relative to the ester bonds. In both cases they use a BMPUS molecule that had a tri-functional silicone head-group. In this work, we only used ester-based initiators but varied the number of bonding units in the silicone head-group. We observed that siloxane bonds are susceptible to hydrolysis, which is likely the main mechanism that leads to the degrafting in the current system. We state so because if the hydrolysis reaction occurred primarily in the ester bond, the extent of degrafting would have been comparable for brushes grown from both mBMPUS and tBMPUS initiators because both initiator molecules possess one ester bond each. We note, however, that hydrolysis in the ester bond can still take place. ${ }^{66,67}$

\section{Discussion}

We employed the stretched exponential function, a continuous sum of exponential decays, to fit our experimental data. ${ }^{68}$ We assume a pseudo first order reaction kinetics with the apparent rate constant $k(t)$,

$$
\begin{aligned}
& k(t)=k_{0} \cdot t^{\beta-1} \\
& \frac{d \sigma}{d t}=-k(t) \cdot \sigma \\
& \sigma=\sigma_{0} \cdot \exp \left(-\frac{k_{0}}{\beta} \cdot t^{\beta}\right)
\end{aligned}
$$

where $k_{0}, t, \beta$, and $\sigma_{0}$ are intrinsic (or initial) rate constant $\left(\mathrm{min}^{-1}\right)$, incubation time (min), a stretching exponent, and the grafting density (chains $/ \mathrm{nm}^{2}$ ), respectively, before incubation in $\mathrm{pH}$ 9 solution. While for $\beta=1$, the rate constant is time independent, for $0<\beta<1$, the exponential function is stretched and $k(t)$ decreases with increasing time. Considering the equilibrium state 
at infinite time without further degrafting, where the grafting density is $\sigma_{e q}$. Equation 4 can be rewritten as:

$$
\sigma=\sigma_{e q}+\left(\sigma_{0}-\sigma_{e q}\right) \cdot \exp \left(-\frac{k_{0}}{\beta} \cdot t^{\beta}\right)
$$

or can be normalized by $\sigma_{N}\left(\right.$ or $h_{N}, c f$. Equation 1):

$$
\frac{\sigma}{\sigma_{N}}=\frac{h}{h_{N}}=\frac{\sigma_{e q}}{\sigma_{N}}+\left(\frac{\sigma_{0}}{\sigma_{N}}-\frac{\sigma_{e q}}{\sigma_{N}}\right) \cdot \exp \left(-\frac{k_{0}}{\beta} \cdot t^{\beta}\right)
$$

For brushes having different DQ values (Figure 7a), $\sigma_{N}$ is equal to $\sigma_{0}$. In the case of the grafting density gradient brushes (Figure 7b), $\sigma_{N}$ represents the highest initial grafting density before TBAF degrafting (i.e., $h_{N} \equiv$ the highest initial dry thickness $=132.0 \mathrm{~nm}$ ).

(a)

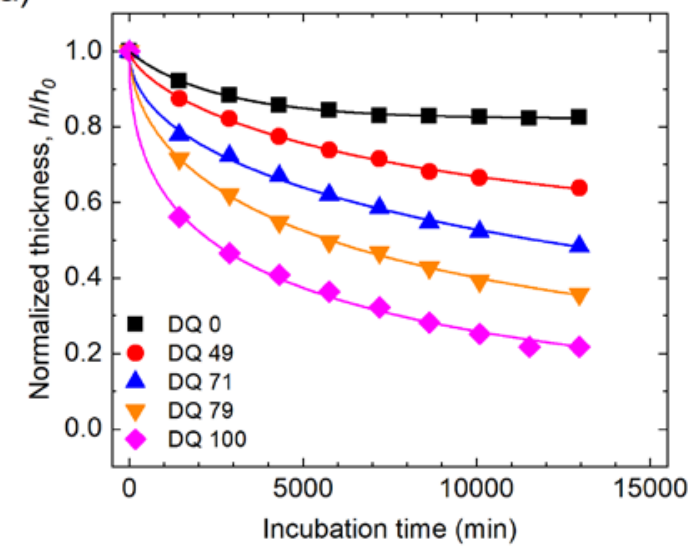

(c)

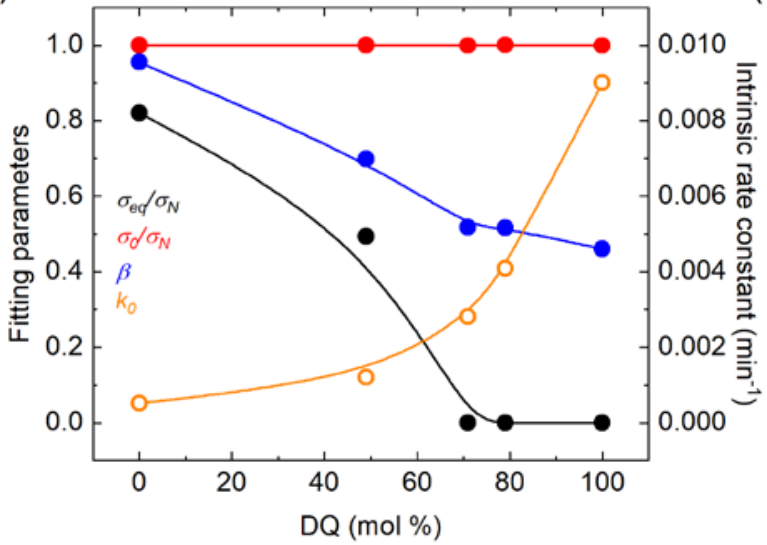

(b)

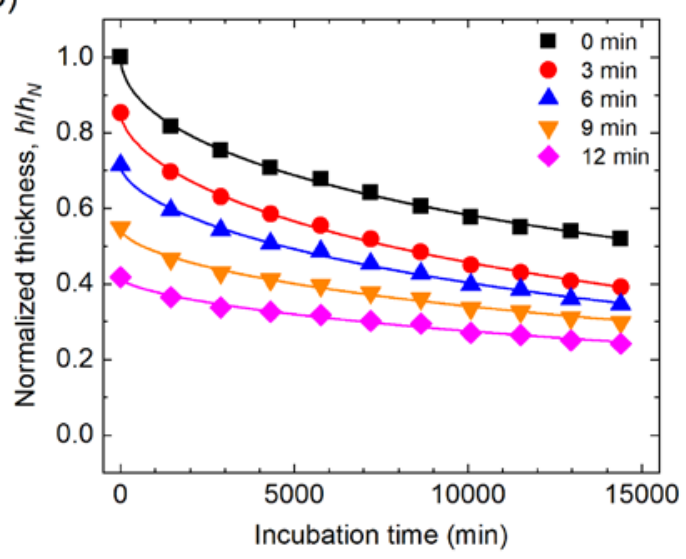

(d)

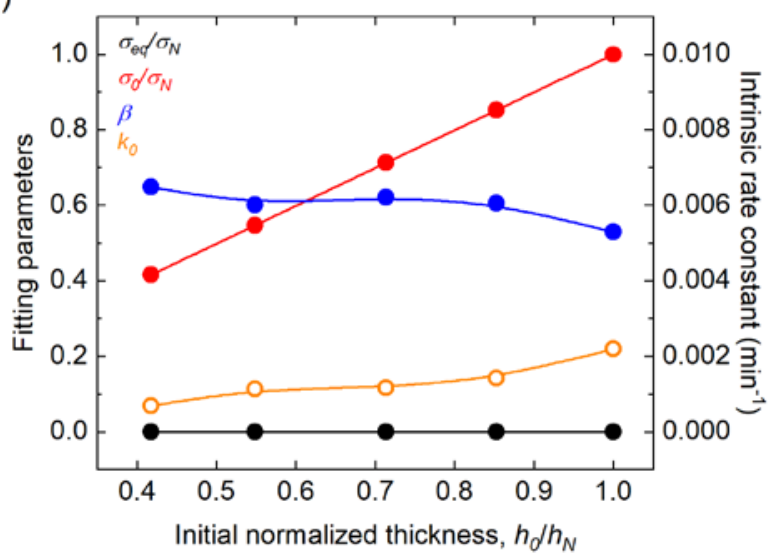

Figure 7. Normalized dry thickness of qPDMAEMA brushes at $100^{\circ} \mathrm{C}$ having (a) different DQ (from Figure 2a) and (b) different grafting density for qPDMAEMA-DQ82 (from Figure 4a). The closed symbol 
depicts experimental data and the corresponding colored lines are the best fits to Equation 6. (c) and (d) display the fitting parameters for (a) and (b), respectively. The values of $k_{0}$ corresponds to the right ordinate in plots (c) and (d). The corresponding colored lines are splines and represent the guide to the eye.

The experimental data (closed symbols) and the best fits (lines) are plotted in Figure 7a and $\mathbf{7 b}$, and the fitting parameters are plotted in Figure 7c and 7d. All data points are fitted with $R^{2}>0.994$. In Figure 7c, all fitted $\sigma_{0} / \sigma_{N}$ values are equal to unity as expected. $\sigma_{e q} / \sigma_{N}$ values decrease with increasing DQ, implying that the grafting densities at equilibrium decrease due to strong mechanical forces that increase with increasing charge density. At the DQ $\geq 71$, the $\sigma_{e q} / \sigma_{N}$ values reach $\sim 0$. Thus, high charge density can remove all the brushes from the substrate. At DQ0, $\beta$ is $\sim 1$, implying that the kinetics resembles an exponential decay. By increasing DQ, the $\beta$ values approach $\sim 0.5$, noting that the apparent rate constant decreases with increasing time. The intrinsic rate constant $k_{0}$ displays a non-linear increase with DQ, supporting the results plotted in Figure 2b. In Figure 7d, $\sigma_{0} / \sigma_{N}$ values are well fitted to the initial normalized thicknesses $\left(h_{0} / h_{N}\right)$. Since all brushes have a high charge density, the $\sigma_{e q} / \sigma_{N}$ values reach values $\sim 0$ at equilibrium. The $\beta$ values are $\sim 0.6$ for nearly all values of $h_{0} / h_{N}$, which further validates the result shown in Figure $\mathbf{4 b}$ and implies that polymer brush degrafting follows the same degrafting mechanism. This is further justified by the master plot in Figure 5. The $k_{0}$ values increase slightly with increasing the grafting density of the polymers.

The degrafting kinetics for qPDMAEMA-DQ82 brushes grown from mBMPUS was also fitted by adopting the same analysis as above (data not shown). $\beta$ was $\sim 0.68$, indicating that the kinetics of degrafting was comparable to that of polymer brushes grown from tBMPUS. This implies that the kinetic of degrafting is independent of the structure of the head-group in the silane initiator. 
Considering all results, we suggest that degrafting of densely-populated polycationic bushes on surfaces changes the temporal grafting density and thus the brush conformation on the substrate. This, in turn, leads to time-dependent reduction of the elastic force that acts on the grafted chains and reduces the extent of degrafting with progressing time. The whole process is characterized by time-dependent and decreasing rate constant describing the degrafting phenomenon.

\section{Conclusions}

We have explored degrafting of weak and strong polycationic brushes based on PDMAEMA and qPDMAEMA, respectively, by incubating them in $\mathrm{pH} 4,7.4$, and 9 solutions at $0.05 \mathrm{M}$ ionic strength with monovalent ions. The extent of degrafting in qPDMAEMA brushes increased with increasing degree of quaternization and incubation time in solution.

The extent of degrafting is the highest in $\mathrm{pH} 9$, supporting the notion that degrafting involves base-catalyzed hydrolysis reaction at the initiator site. Increasing the degree of dissociation (due to increased $\mathrm{pH}$ and/or increased DQ) increases brush swelling, in turn, the increases elastic force applied on the initiators. This results in severe degrafting of the brushes from the substrates. The degrafting rate increases with increasing the grafting density of polymer brushes (Figure 4a). The degrafting mechanism is the same for all polymer brush systems studied, as revealed from the data in Figures 4b, 5, and 7. It involves time-dependent variation in the elastic force acting on grafted chains that decreases with increasing time and gives rise to time-depended conformations of polymer grafts on the substrate. Interestingly, we observed that the degrafting process depended on the molecular weight of qPDMAEMA brushes. Specifically, we observed higher degrafting with increasing MW of the polymers and DQ. While not predicted by the theory, we tentatively 
explained the behavior by considering molecular weight dispersity of the brushes that may affect the instantaneous conformation of polymer chains on the substrate and thus the local elastic force. By comparing the degrafting of polymer brushes from tBMPUS and mBMPUS, we concluded the polymer brush degrafting took place primarily at the siloxane head-group of the initiator that held the chains attached to the substrate.

\section{Associated content}

\section{Supporting information}

The Supporting Information is available free of charge on the ACS Publications website.

Details pertaining to preparation and characterization of polymer films; dry thicknesses of PDMAEMA brushes with MW gradient and grafting density gradient; FTIR spectra of tested polycationic brushes (PDF)

\section{Author information}

*Email: jgenzer@ncsu.edu

ORCID: Yeongun Ko: 0000-0001-5770-6707

ORCID: Jan Genzer: 0000-0002-1633-238X

\section{Acknowledgement}

The work was supported by the National Science Foundation, Grant no. DMR-1404639. 


\section{References}

(1) Barbey, R.; Lavanant, L.; Paripovic, D.; Schüwer, N.; Sugnaux, C.; Tugulu, S.; Klok, H. A. Polymer Brushes via Surface-Initiated Controlled Radical Polymerization: Synthesis, Characterization, Properties, and Applications. Chem. Rev. 2009, 109, 5437-5527.

(2) Chen, W. L.; Cordero, R.; Tran, H.; Ober, C. K. 50th Anniversary Perspective: Polymer Brushes: Novel Surfaces for Future Materials. Macromolecules 2017, 50, 4089-4113.

(3) Zoppe, J. O.; Ataman, N. C.; Mocny, P.; Wang, J.; Moraes, J.; Klok, H. A. SurfaceInitiated Controlled Radical Polymerization: State-of-the-Art, Opportunities, and Challenges in Surface and Interface Engineering with Polymer Brushes. Chem. Rev. 2017, 117, 1105-1318.

(4) Brittain, W. J.; Minko, S. A Structural Definition of Polymer Brushes. J. Polym. Sci. Part A Polym. Chem. 2007, 45, 3505-3512.

(5) Alexander, S. Adsorption of Chain Molecules with a Polar Head a Scaling Description. J. Phys. 1977, 38, 983-987.

(6) de Gennes, P. G. Conformations of Polymers Attached to an Interface. Macromolecules 1980, 13, 1069-1075.

(7) Milner, S. T.; Witten, T. A.; Cates, M. E. A Parabolic Density Profile for Grafted Polymers. Epl 1988, 5, 413-418.

(8) Milner, S. T. Polymer Brushes. Science (80-. ). 1991, 251, 905-914.

(9) Lai, P.-Y.; Zhulina, E. B. Monte Carlo Test of the Self-Consistent Field Theory of a Polymer Brush. J. Phys. II 1992, 2, 547-560.

(10) Netz, R. R.; Schick, M. Polymer Brushes: From Self-Consistent Field Theory to Classical Theory. Macromolecules 1998, 31, 5105-5122.

(11) Azzaroni, O. Polymer Brushes Here, There, and Everywhere: Recent Advances in Their Practical Applications and Emerging Opportunities in Multiple Research Fields. J. Polym. Sci. Part A Polym. Chem. 2012, 50, 3225-3258.

(12) Kreer, T. Polymer-Brush Lubrication: A Review of Recent Theoretical Advances. Soft Matter 2016, 12, 3479-3501.

(13) Giussi, J. M.; Cortez, M. L.; Marmisollé, W. A.; Azzaroni, O. Practical Use of Polymer Brushes in Sustainable Energy Applications: Interfacial Nanoarchitectonics for HighEfficiency Devices. Chem. Soc. Rev. 2019, 48, 814-849.

(14) Tran, Y.; Auroy, P. Synthesis of Poly(Styrene Sulfonate) Brushes. J. Am. Chem. Soc. 2001, 123, 3644-3654.

(15) Biesalski, M.; Rühe, J. Scaling Laws for the Swelling of Neutral and Charged Polymer Brushes in Good Solvents. Macromolecules 2002, 35, 499-507.

(16) Wu, T.; Efimenko, K.; Vlěek, P.; Šubr, V.; Genzer, J. Formation and Properties of Anchored Polymers with a Gradual Variation of Grafting Densities on Flat Substrates. Macromolecules 2003, 36, 2448-2453.

(17) Sanjuan, S.; Perrin, P.; Pantoustier, N.; Tran, Y. Synthesis and Swelling Behavior of PH- 
Responsive Polybase Brushes. Langmuir 2007, 23, 5769-5778.

(18) Wu, T.; Gong, P.; Szleifer, I.; Vlček, P.; Šubr, V.; Genzer, J. Behavior of SurfaceAnchored Poly(Acrylic Acid) Brushes with Grafting Density Gradients on Solid Substrates: 1. Experiment. Macromolecules 2007, 40, 8756-8764.

(19) Wu, T.; Gong, P.; Szleifer, I.; Vlček, P.; Šubr, V.; Genzer, J. Behavior of SurfaceAnchored Poly(Acrylic Acid) Brushes with Grafting Density Gradients on Solid Substrates: 1. Experiment. Macromolecules 2007, 40, 8756-8764.

(20) Riachi, C.; Sch, N.; Klok, H. Degradable Polymer Brushes Prepared via Surface-Initiated Controlled Radical Polymerization. Macromolecules 2009, 42, 8076-8081.

(21) Tugulu, S.; Klok, H. A. Stability and Nonfouling Properties of Poly(Poly(Ethylene Glycol) Methacrylate) Brushes under Cell Culture Conditions. Biomacromolecules 2008, 9, 906-912.

(22) Wang, J.; Klok, H.-A. Swelling-Induced Chain Stretching Enhances Hydrolytic Degrafting of Hydrophobic Polymer Brushes in Organic Media. Angew. Chemie Int. Ed. 2019, 0 .

(23) Klok, H.-A.; Genzer, J. Expanding the Polymer Mechanochemistry Toolbox through Surface-Initiated Polymerization. ACS Macro Lett. 2015, 4, 636-639.

(24) Roling, O.; De Bruycker, K.; Vonhören, B.; Stricker, L.; Körsgen, M.; Arlinghaus, H. F.; Ravoo, B. J.; Du Prez, F. E. Rewritable Polymer Brush Micropatterns Grafted by Triazolinedione Click Chemistry. Angew. Chemie Int. Ed. 2015, n/a-n/a.

(25) Kang, C.; Crockett, R. M.; Spencer, N. D. Molecular-Weight Determination of Polymer Brushes Generated by SI-ATRP on Flat Surfaces. Macromolecules 2014, 47, 269-275.

(26) Xiong, X.; Xue, L.; Cui, J. Phototriggered Growth and Detachment of Polymer Brushes with Wavelength Selectivity. ACS Macro Lett. 2018, 7, 239-243.

(27) Wei, W.; Balamurugan, A.; Dwyer, J. H.; Gopalan, P. Substrate-Independent Approach to Dense Cleavable Polymer Brushes by Nitroxide-Mediated Polymerization. ACS Macro Lett. 2018, 7, 100-104.

(28) Li, Y.; Niu, Z.; Burdyńska, J.; Nese, A.; Zhou, Y.; Kean, Z. S.; Dobrynin, A. V.; Matyjaszewski, K.; Craig, S. L.; Sheiko, S. S. Sonication-Induced Scission of Molecular Bottlebrushes: Implications of the "Hairy" Architecture. Polymer (Guildf). 2016, 84, 178184.

(29) Schaefer, M.; Icli, B.; Weder, C.; Lattuada, M.; Kilbinger, A. F. M.; Simon, Y. C. The Role of Mass and Length in the Sonochemistry of Polymers. Macromolecules 2016, 49, 1630-1636.

(30) von Werne, T.; Patten, T. E. Atom Transfer Radical Polymerization from Nanoparticles: A Tool for the Preparation of Well-Defined Hybrid Nanostructures and for Understanding the Chemistry of Controlled/"living" Radical Polymerizations from Surfaces. J. Am. Chem. Soc. 2001, 123, 7497-7505.

(31) Koylu, D.; Carter, K. R. Stimuli-Responsive Surfaces Utilizing Cleavable Polymer Brush Layers. Macromolecules 2009, 42, 8655-8660. 
(32) Hansson, S.; Antoni, P.; Bergenudd, H.; Malmström, E. Selective Cleavage of Polymer Grafts from Solid Surfaces: Assessment of Initiator Content and Polymer Characteristics. Polym. Chem. 2011, 2, 556-558.

(33) Melzak, K. A.; Yu, K.; Bo, D.; Kizhakkedathu, J. N.; Toca-Herrera, J. L. Chain Length and Grafting Density Dependent Enhancement in the Hydrolysis of Ester-Linked Polymer Brushes. Langmuir 2015, 31, 6463-6470.

(34) S.S., S.; F.C., S.; A., R.; D., S.; M., R.; H.-I., L.; K., M. Adsorption-Induced Scission of Carbon-Carbon Bonds. Nature 2006, 440, 191-194.

(35) Lyu, B.; Cha, W.; Mao, T.; Wu, Y.; Qian, H.; Zhou, Y.; Chen, X.; Zhang, S.; Liu, L.; Yang, G.; et al. Surface Confined Retro Diels-Alder Reaction Driven by the Swelling of Weak Polyelectrolytes. ACS Appl. Mater. Interfaces 2015, 7, 6254-6259.

(36) Kopyshev, A.; Galvin, C. J.; Genzer, J.; Lomadze, N.; Santer, S. Opto-Mechanical Scission of Polymer Chains in Photosensitive Diblock-Copolymer Brushes. Langmuir 2013, 29, 13967-13974.

(37) Zhang, Y.; He, J.; Zhu, Y.; Chen, H.; Ma, H. Directly Observed Au-S Bond Breakage Due to Swelling of the Anchored Polyelectrolyte. Chem. Commun. 2011, 47, 1190-1192.

(38) Patil, R. R.; Turgman-Cohen, S.; Šrogl, J.; Kiserow, D.; Genzer, J. On-Demand Degrafting and the Study of Molecular Weight and Grafting Density of Poly(Methyl Methacrylate) Brushes on Flat Silica Substrates. Langmuir 2015, 31, 2372-2381.

(39) Patil, R. R.; Turgman-Cohen, S.; Šrogl, J.; Kiserow, D.; Genzer, J. Direct Measurement of Molecular Weight and Grafting Density by Controlled and Quantitative Degrafting of Surface-Anchored Poly(Methyl Methacrylate). ACS Macro Lett. 2015, 4, 251-254.

(40) Miles, J.; Schlenker, S.; Ko, Y.; Patil, R.; Rao, B. M.; Genzer, J. Design and Fabrication of Wettability Gradients with Tunable Profiles through Degrafting Organosilane Layers from Silica Surfaces by Tetrabutylammonium Fluoride. Langmuir 2017, 33, 1455614564.

(41) Patil, R.; Miles, J.; Ko, Y.; Datta, P.; Rao, B. M.; Kiserow, D.; Genzer, J. Kinetic Study of Degrafting Poly(Methyl Methacrylate) Brushes from Flat Substrates by Tetrabutylammonium Fluoride. Macromolecules 2018, 51, 10237-10245.

(42) Bain, E. D.; Dawes, K.; Özçam, A. E.; Hu, X.; Gorman, C. B.; Šrogl, J.; Genzer, J. Surface-Initiated Polymerization by Means of Novel, Stable, Non-Ester-Based Radical Initiator. Macromolecules 2012, 45, 3802-3815.

(43) Galvin, C. J.; Bain, E. D.; Henke, A.; Genzer, J. Instability of Surface-Grafted Weak Polyacid Brushes on Flat Substrates. Macromolecules 2015, 48, 5677-5687.

(44) Li, Y.; Ko, Y.; Lin, Y.; Kiserow, D.; Genzer, J. Enhanced Stability of Surface-Tethered Diblock Copolymer Brushes with a Neutral Polymer Block and a Weak Polyelectrolyte Block: Effects of Molecular Weight and Hydrophobicity of the Neutral Block. Macromolecules 2017, 50, 8580-8587.

(45) Li, Y.; Lin, Y.; Ko, Y.; Kiserow, D.; Genzer, J. Visualization of MechanochemicallyAssisted Degrafting of Surface-Tethered Poly(Acrylic Acid) Brushes. ACS Macro Lett. 2018, 7, 609-613. 
(46) Ataman, N. C.; Klok, H.-A. Degrafting of Poly(Poly(Ethylene Glycol) Methacrylate) Brushes from Planar and Spherical Silicon Substrates. Macromolecules 2016, 49, 90359047.

(47) Aden, B.; Kite, C. M.; Hopkins, B. W.; Zetterberg, A.; Lokitz, B. S.; Ankner, J. F.; Kilbey, S. M. Assessing Chemical Transformation of Reactive, Interfacial Thin Films Made of End-Tethered Poly(2-Vinyl-4,4-Dimethyl Azlactone) (PVDMA) Chains. Macromolecules 2017, 50, 618-630.

(48) Chen, W. L.; Menzel, M.; Prucker, O.; Wang, E.; Ober, C. K.; Rühe, J. Morphology of Nanostructured Polymer Brushes Dependent on Production and Treatment. Macromolecules 2017, 50, 4715-4724.

(49) Chen, W. L.; Menzel, M.; Watanabe, T.; Prucker, O.; Rühe, J.; Ober, C. K. Reduced Lateral Confinement and Its Effect on Stability in Patterned Strong Polyelectrolyte Brushes. Langmuir 2017, 33, 3296-3303.

(50) Yu, Y.; Vancso, G. J.; de Beer, S. Substantially Enhanced Stability against Degrafting of Zwitterionic PMPC Brushes by Utilizing PGMA-Linked Initiators. Eur. Polym. J. 2017, 89, 221-229.

(51) Enomoto, K.; Takahashi, S.; Iwase, T.; Yamashita, T.; Maekawa, Y. Degradation Manner of Polymer Grafts Chemically Attached on Thermally Stable Polymer Films: SwellingInduced Detachment of Hydrophilic Grafts from Hydrophobic Polymer Substrates in Aqueous Media. J. Mater. Chem. 2011, 21, 9343-9349.

(52) Liberelle, B.; Giasson, S. Chemical End-Grafting of Homogeneous Polystyrene Monolayers on Mica and Silica Surfaces. Langmuir 2007, 23, 9263-9270.

(53) Ko, Y.; Miles, J.; Genzer, J. Contraction/Expansion and Moisture Uptake/Retention during Thermal Treatment of Thin Hydrophilic Polymer Films. Submitted.

(54) Nová, L.; Uhlík, F.; Košovan, P. Local PH and Effective PKA of Weak PolyelectrolytesInsights from Computer Simulations. Phys. Chem. Chem. Phys. 2017, 19, 14376-14387.

(55) Nap, R.; Gong, P.; Szleifer, I. Weak Polyelectrolytes Tethered to Surfaces: Effect of Geometry, Acid-Base Equilibrium and Electrical Permittivity. J. Polym. Sci. Part B Polym. Phys. 2006, 44, 2638-2662.

(56) Van De Wetering, P.; Moret, E. E.; Schuurmans-Nieuwenbroek, N. M. E.; Van Steenbergen, M. J.; Hennink, W. E. Structure-Activity Relationships of Water-Soluble Cationic Methacrylate/Methacrylamide Polymers for Nonviral Gene Delivery. Bioconjug. Chem. 1999, 10, 589-597.

(57) Dong, R.; Lindau, M.; Ober, C. K. Dissociation Behavior of Weak Polyelectrolyte Brushes on a Planar Surface. Langmuir 2009, 25, 4774-4779.

(58) Milner, S. T.; Witten, T. A.; Cates, M. E. Effects of Polydispersity in the End-Grafted Polymer Brush. Macromolecules 1989, 22, 853-861.

(59) Qi, S.; Klushin, L. I.; Skvortsov, A. M.; Schmid, F. Polydisperse Polymer Brushes: Internal Structure, Critical Behavior, and Interaction with Flow. Macromolecules 2016, 49, 9665-9683. 
(60) Okrugin, B. M.; Richter, R. P.; Leermakers, F. A. M.; Neelov, I. M.; Borisov, O. V.; Zhulina, E. B. Structure and Properties of Polydisperse Polyelectrolyte Brushes Studied by Self-Consistent Field Theory. Soft Matter 2018, 14, 6230-6242.

(61) Kang, C.; Crockett, R.; Spencer, N. D. The Influence of Surface Grafting on the Growth Rate of Polymer Chains. Polym. Chem. 2016, 7, 302-309.

(62) Turgman-Cohen, S.; Genzer, J. Simultaneous Bulk- and Surface-Initiated Controlled Radical Polymerization from Planar Substrates. J. Am. Chem. Soc. 2011, 133, 1756717569.

(63) Genzer, J.; Efimenko, K.; Fischer, D. A. Molecular Orientation and Grafting Density in Semifluorinated Self-Assembled Monolayers of Mono-, Di-, and Trichloro Silanes on Silica Substrates. Langmuir 2002, 18, 9307-9311.

(64) Genzer, J.; Efimenko, K.; Fischer, D. A. Formation Mechanisms and Properties of Semifluorinated Molecular Gradients on Silica Surfaces. Langmuir 2006, 22, 8532-8541.

(65) Klok, H. A.; Genzer, J. Expanding the Polymer Mechanochemistry Toolbox through Surface-Initiated Polymerization. ACS Macro Lett. 2015, 4, 636-639.

(66) Bain, E. D.; Dawes, K.; Özçam, A. E.; Hu, X.; Gorman, C. B.; Šogl, J.; Genzer, J. Surface-Initiated Polymerization by Means of Novel, Stable, Non-Ester-Based Radical Initiator. Macromolecules 2012, 45, 3802-3815.

(67) Galvin, C. J.; Bain, E. D.; Henke, A.; Genzer, J. Instability of Surface-Grafted Weak Polyacid Brushes on Flat Substrates. Macromolecules 2015, 48, 5677-5687.

(68) Williams, G. Molecular Aspects of Multiple Dielectric Relaxation Processes in Solid Polymers. In Electric Phenomena in Polymer Science; Springer Berlin Heidelberg: Berlin, Heidelberg, 2008; pp 59-92. 
TOC figure

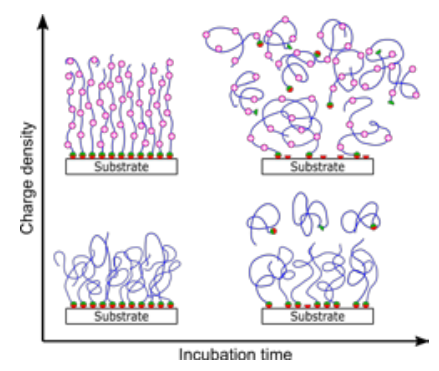

Larger version of the TOC figure

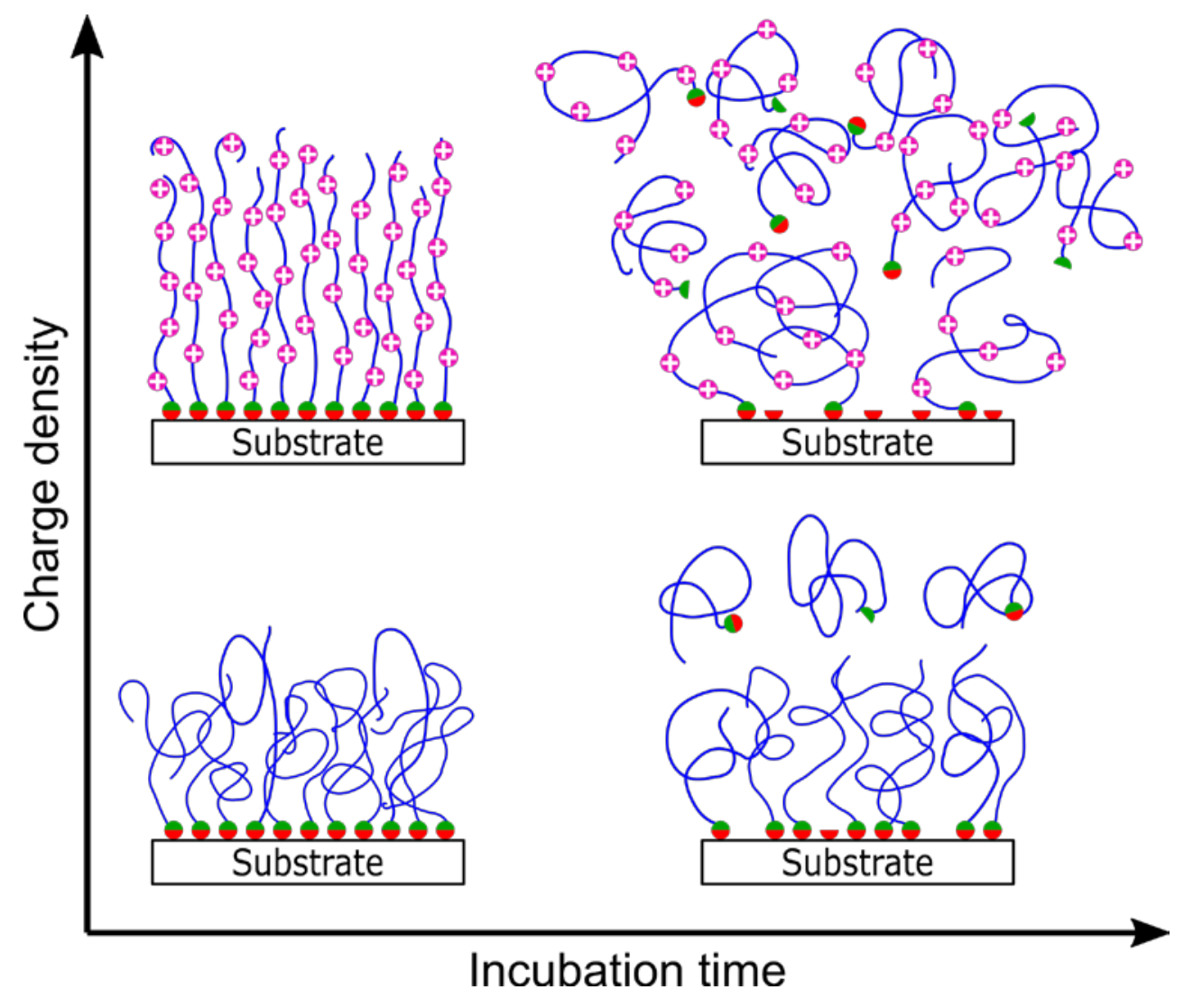




\title{
Supporting Information
}

Spontaneous degrafting of weak and strong polycationic brushes in aqueous buffer solutions

\author{
Yeongun $\mathrm{Ko}^{1}$, Jan Genzer ${ }^{1,2, *}$ \\ ${ }^{1}$ Department of Chemical and Biomolecular Engineering, North Carolina State University \\ Raleigh, NC 27695-7905, USA \\ ${ }^{2}$ Global Station for Soft Matter, Global Institution for Collaborative Research and Education \\ (GI-CoRE), Hokkaido University, Japan
}

\section{Background about scaling concepts of polymer brush height}

The wet thickness $H$ of neutral polymer brushes in a good solvent is given by:

$$
H_{N B} \propto N b^{5 / 3} \sigma^{1 / 3}
$$

where $b$ represents size of the repeat units. This relationship results from the balance between the osmotic pressure and entropic force acting on the grafted polymers. ${ }^{1}$ The equilibrium state of charged brushes is determined by the balance between the electrostatic interactions of charged monomers and osmotic pressure due to counter ions. ${ }^{2,3}$ Thus, the chain conformation depends on the concentration of counter ions and external salt. At low salt concentrations, most counter ions are confined inside the wet brush to neutralize the charges. In this, so-called osmotic brush (OsB) regime, the wet thickness $H$ of weak polyelectrolyte brushes is given by:

$$
H_{O S B, w e a k} \propto N b^{4 / 3} \sigma^{-1 / 3}\left[\left(\frac{\alpha_{b}}{1-\alpha_{b}}\right)\left(C_{\mathrm{H}^{+}}+C_{S}\right)\right]^{1 / 3}
$$

where $\alpha_{b}, C_{\mathrm{H}^{+}}$and $C_{S}$ are the bulk degree of dissociation, proton and external monovalent salt molar concentration, respectively. The wet thickness depends on the polymer grafting density and the concentration of external salt. For strong polyelectrolyte brushes, the wet thickness in the OsB regime is given by:

$$
H_{O S B, \text { strong }} \propto N b \alpha_{b}{ }^{1 / 2}
$$

\footnotetext{
*Corresponding author: jgenzer@ncsu.edu
} 
The brush height does not depend on the grafting density or external salt concentration. With increasing salt concentration, the charged units start to be screened and the brushes contract. In this, so-called salted brush (SB) regime, the brush height of both weak and strong polyelectrolyte brushes possesses the same scaling relation:

$$
H_{S B} \propto N b^{2 / 3} \sigma^{1 / 3} \alpha_{b}{ }^{2 / 3} C_{S}{ }^{-1 / 3}
$$

The wet thickness in the SB regime decreases with increasing salt concentration. Importantly, the wet thickness of both weak and strong polyelectrolyte brushes increases with increasing the degree of dissociation. The border line between the OsB and SB regimes is determined by the external salt volume fraction $\varphi_{S} \propto \sigma \cdot\left(\alpha_{b}\right)^{1 / 2}$.

The ionic strength in our experiments was kept at $0.05 \mathrm{M}$, which leads to the assumption that the brushes were in OsB regime with $\sigma=0.5$ chains $/ \mathrm{nm}^{2}$. When swollen, the polymer chains lose their configurational entropy and are under the influence of a strong elastic force $\left(f_{\text {entropic }}\right)$ :

$$
f_{\text {entropic }} \propto \frac{k_{B} T}{b}\left(1-\frac{H}{N \cdot b}\right)^{-1}
$$

where $k_{B}$ and $T$ are Boltzmann constant and the absolute temperature, respectively. ${ }^{4}$ At weak stretching $(H \ll N \cdot b)$, the elastic force is estimated from the Hook's law. When the brushes are strongly stretched $(H \leq N \cdot b)$, the elastic force increases nonlinearly with increasing $H$.

The swelling ratio, defined as the wet thickness normalized by the dry thickness, gives rise to the following relationships:

$$
\begin{aligned}
& \frac{H_{N B}}{h} \propto N^{0} b^{-4 / 3} \sigma^{-2 / 3} \\
& \frac{H_{\text {OSB,weak }}}{h} \propto N^{0} b^{-5 / 3} \sigma^{-4 / 3}\left[\left(\frac{\alpha_{b}}{1-\alpha_{b}}\right)\left(C_{\mathrm{H}^{+}}+C_{S}\right)\right]^{1 / 3} \\
& \frac{H_{\text {OSB,strong }}}{h} \propto N^{0} b^{-2} \sigma^{-1} \alpha_{b}{ }^{1 / 2} \\
& \frac{H_{S B}}{h} \propto N^{0} b^{-7 / 3} \sigma^{-2 / 3} \alpha_{b}{ }^{2 / 3} C_{S}{ }^{-1 / 3}
\end{aligned}
$$


(a)

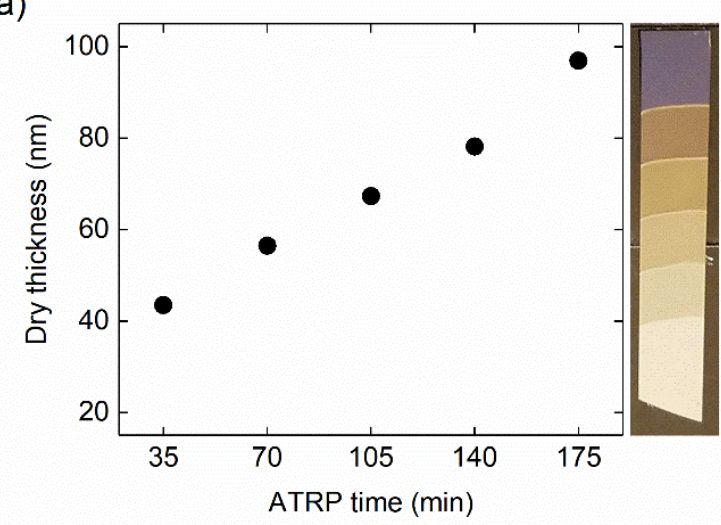

(b)

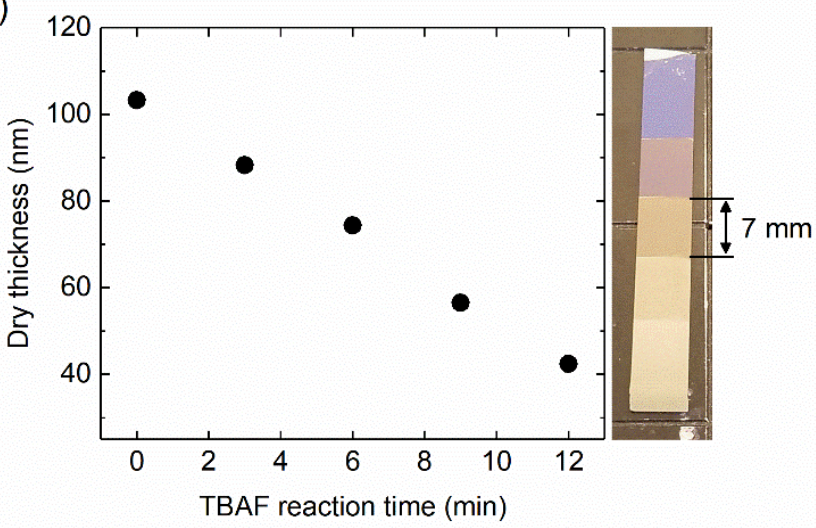

Figure S1. Dry thicknesses of PDMAEMA brushes measured at $100^{\circ} \mathrm{C}$ with (a) varying ATRP polymerization time and (b) gradually-degrafted initiator with various TBAF times following by the ATRP reaction for $120 \mathrm{~min}$. The images on the right of each plot depict the photographs of the actual samples.

The samples featuring the molecular weight or grafting density gradients of PDMAEMA brushes were fabricated by a vertical stepwise dipping manor into an atom transfer radical polymerization (ATRP) solution or tetrabutylammonium fluoride (TBAF) solution, respectively. To form gradients in molecular weight, the tBMPUS deposited silicon substrate $(1.4 \mathrm{~cm} \mathrm{x} 4 \mathrm{~cm})$ has been dipped vertically and stepwise into an ATRP solution every $7 \mathrm{~mm}$ for 35 minutes. The plot in Figure S1a exhibits linearly increasing dry thicknesses with polymerization time. Since the substrate has a constant grafting density along the gradient, the increasing dry thicknesses indicate increasing degree of polymerization. This claim can be supported by swelling ratio values in Figure 3a. To form a grafting density gradient of polymer brushes, the substrate featuring a homogeneous tBMPUS layer on a silicon substrate has been degrafted by vertically stepwise dipping into a $0.1 \mathrm{M}$ TBAF solution in dimethylacetamide at $50^{\circ} \mathrm{C}(7 \mathrm{~mm}$ dip each $3 \mathrm{~min}) .{ }^{5}$ During the incubation, the TBAF selectively cleaved siloxane bonds and removed the silanes on the substrate. ${ }^{6,7}$ The substrate was subsequently immersed into an ATRP solution for 120 minutes at room temperature to obtain grafting density gradient polymer brushes. The decrease of dry thicknesses in Figure S1b reflects decreasing grafting densities under the assumption that the molecular weight of all brushes remained the same along the substrate. 


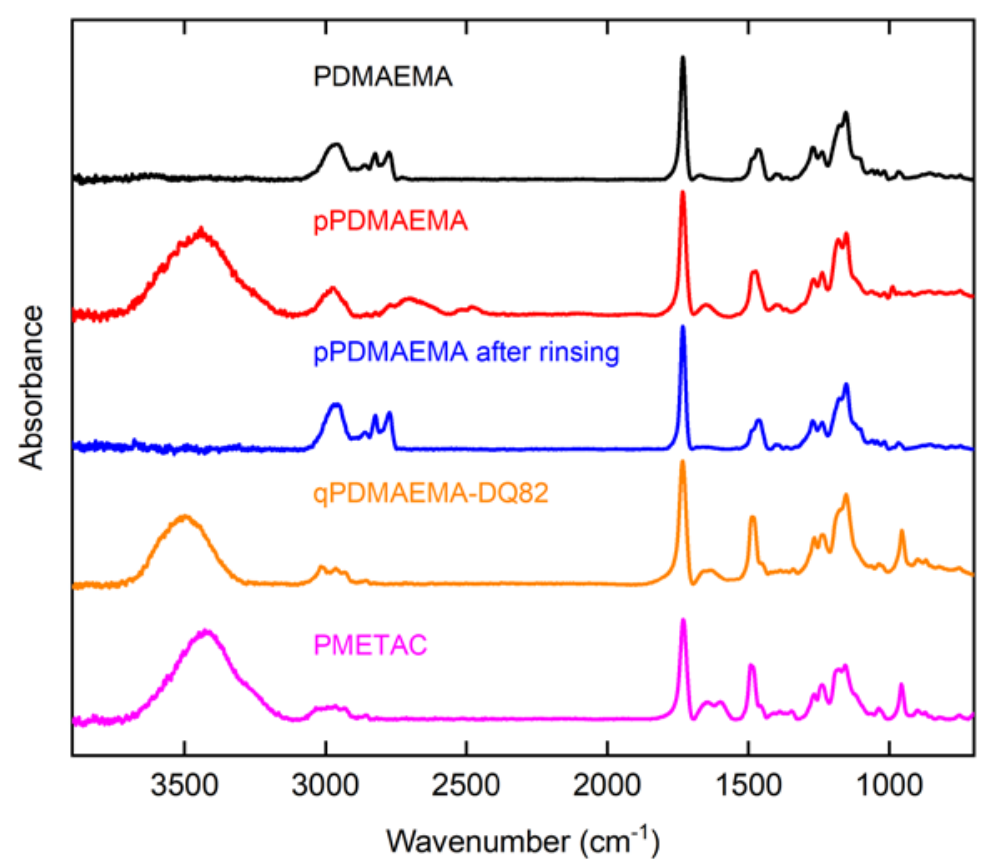

Figure S2. FTIR-ATR spectra for different polymer brushes. All polymer films have comparable dry thickness as $100 \mathrm{~nm}$ and all spectra absorbance are normalized by the intensity of carbonyl peak, 1723 $\mathrm{cm}^{-1}$. The protonated PDMAEMA (pPDMAEMA, red spectra) brushes are acquired after incubating $\mathrm{pH} 4$ solution for 10 mins.

Fourier-transform infrared spectroscopy (FTIR) spectra were collected in attenuated total reflection (ATR) mode with Ge crystal on a Nicolet 6700 spectrometer and analyzed with OMNIC software. All spectra were collected by performing 128 scans with $4 \mathrm{~cm}^{-1}$ resolution after ATR correction followed by manual baseline correction. The spectra of pristine PDMAEMA brushes show 2770 and $2820 \mathrm{~cm}^{-1}$ signals which correspond to asymmetric and symmetric C-H stretching vibrations corresponding to $-\mathrm{N}\left(\mathrm{CH}_{3}\right)_{2}$, respectively. ${ }^{8}$ After 10 min incubation of PDMAEMA brushes in $\mathrm{pH} 4$ solution, most repeat units are protonated and the characteristic C-H signals disappear. A broad band around $3450 \mathrm{~cm}^{-1}$ in the protonated PDMAEMA (pPDMAEMA) is attributed to strong water sorption in addition to $\mathrm{N}-\mathrm{H}$ stretching. After rigorous rinsing of pPDMAEMA by methanol and deionized water, the protonation is suppressed and the IR spectrum is identical to that of the original PDMAEMA. Quaternization with methyl iodide suppresses the C-H vibrations at 2770 and $2820 \mathrm{~cm}^{-1}$ in qPDMAEMA-DQ82. The intensities of the 953 and 1477 $\mathrm{cm}^{-1}$ signals noticeably increase, indicating $\mathrm{N}^{+}-\mathrm{C}$ stretching vibrations. ${ }^{9-11}$ The broad band appears at $\sim 3500 \mathrm{~cm}^{-1}$, which is different from that in the qPDMAEMA spectra. PMETAC shows very similar spectra to qPDMAEMA-DQ82 except for a slight shift of the broad peak at $3420 \mathrm{~cm}^{-1}$. 


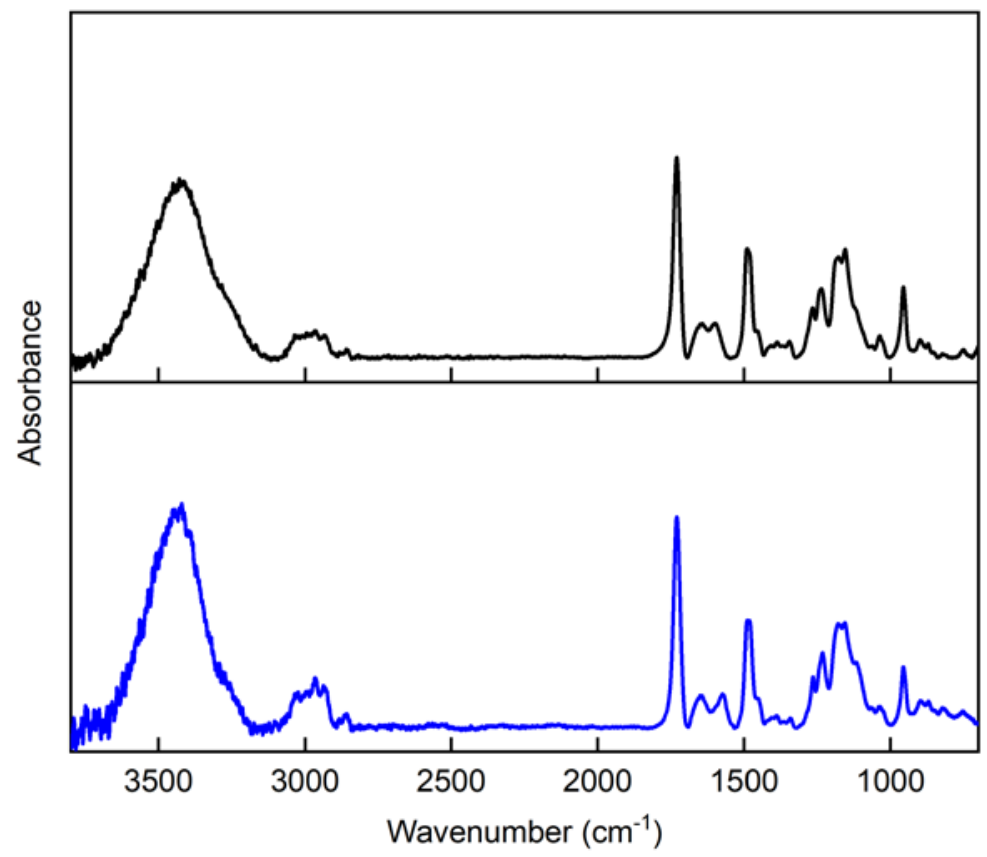

Figure S3. FTIR-ATR spectra for PMETAC brushes as prepared (top, dry thickness $101 \mathrm{~nm}$ ) and after incubating for 2 days in $\mathrm{pH} 9$ solution (bottom, dry thickness $47 \mathrm{~nm}$ ).

To support our claim that degrafting occurs at the initiator site, the FTIR-ATR spectra have been collected before and after degrafting of PMETAC brushes from the substrate. After 2 days of incubation, the dry thickness decreased from $101 \mathrm{~nm}$ to $47 \mathrm{~nm}$. If the repeat units of the polymers were hydrolyzed, the peak intensity of 953 and $1477 \mathrm{~cm}^{-1}$ would have reduced after degrafting. Both IR spectra in Figure S3 are nearly identical indicating that trans-esterification in the repeat units is negligible during the sample incubation. 


\section{References}

(1) Chen, W. L.; Cordero, R.; Tran, H.; Ober, C. K. 50th Anniversary Perspective: Polymer Brushes: Novel Surfaces for Future Materials. Macromolecules 2017, 50, 4089-4113.

(2) Israëls, R.; Leermakers, F. A. M.; Fleer, G. J.; Zhulina, E. B. Charged Polymeric Brushes: Structure and Scaling Relations. Macromolecules 1994, 27, 3249-3261.

(3) Sanjuan, S.; Perrin, P.; Pantoustier, N.; Tran, Y. Synthesis and Swelling Behavior of PHResponsive Polybase Brushes. Langmuir 2007, 23, 5769-5778.

(4) Janshoff, A.; Neitzert, M.; Oberdörfer, Y.; Fuchs, H. Force Spectroscopy of Molecular Systems-Single Molecule Spectroscopy of Polymers and Biomolecules. Angew. Chemie Int. Ed. 2000, 39, 3212-3237.

(5) Miles, J.; Schlenker, S.; Ko, Y.; Patil, R.; Rao, B. M.; Genzer, J. Design and Fabrication of Wettability Gradients with Tunable Profiles through Degrafting Organosilane Layers from Silica Surfaces by Tetrabutylammonium Fluoride. Langmuir 2017, 33, 1455614564.

(6) Patil, R. R.; Turgman-Cohen, S.; Šrogl, J.; Kiserow, D.; Genzer, J. On-Demand Degrafting and the Study of Molecular Weight and Grafting Density of Poly(Methyl Methacrylate) Brushes on Flat Silica Substrates. Langmuir 2015, 31, 2372-2381.

(7) Patil, R. R.; Turgman-Cohen, S.; Šrogl, J.; Kiserow, D.; Genzer, J. Direct Measurement of Molecular Weight and Grafting Density by Controlled and Quantitative Degrafting of Surface-Anchored Poly(Methyl Methacrylate). ACS Macro Lett. 2015, 4, 251-254.

(8) Özçam, A. E.; Roskov, K. E.; Spontak, R. J.; Genzer, J. Generation of Functional PET Microfibers through Surface-Initiated Polymerization. J. Mater. Chem. 2012, 22, 58555864.

(9) Donegan, M.; Dowling, D. P. Protein Adhesion on Atmospheric Plasma Deposited Quaternary Ammonium Salt Coatings. Plasma Process. Polym. 2013, 10, 526-534.

(10) Badawy, M. E. I.; Rabea, E. I.; Taktak, N. E. M. Antimicrobial and Inhibitory Enzyme Activity of N-(Benzyl) and Quaternary N-(Benzyl) Chitosan Derivatives on Plant Pathogens. Carbohydr. Polym. 2014, 111, 670-682.

(11) Hu, X.; Lin, X.; Zhao, H.; Chen, Z.; Yang, J.; Li, F.; Liu, C.; Tian, F. Surface Functionalization of Polyethersulfone Membrane with Quaternary Ammonium Salts for Contact-Active Antibacterial and Anti-Biofouling Properties. Materials 2016, 9, 376-387. 